\title{
Universal RNA editing in a human liver at the fetal stage
}

\author{
Dong Liu ${ }^{1}$, Cong Liu ${ }^{1}$, Xiyin Wang ${ }^{1}$, Sigurdur Ingvarsson ${ }^{2}$, Huiping Chen ${ }^{1}$ \\ ${ }^{1}$ Department of Medical Genetics, Tongji Medical College, Huazhong University of Science and Technology, Wuhan, China \\ ${ }^{2}$ Institute for Experimental Pathology and Faculty of Medicine, University of Iceland, Keldur, Iceland \\ Email: huiping@mail.hust.edu.cn
}

Received 14 May 2012; revised 17 June 2012; accepted 10 July 2012

\begin{abstract}
It is known that RNA editing occurs in human cells, which can change the information transmission from DNA to RNA and proteins. Most previous studies have focused on editing of the mRNAs. Here we reported that several kinds of RNAs, including miRNA, rRNA, mRNA, miscRNA and unknown RNA, exhibited base editing in a human fetal liver. Several editing types are displayed. Our data reveals that RNA editing may occur in different species of RNAs.
\end{abstract}

Keywords: RNA Editing; miRNA; rRNA; mRNA; miscRNA; Fetal Liver

\section{INTRODUCTION}

The central dogma of molecular biology is that "DNA makes RNA makes protein" or "RNA makes DNA makes RNA makes protein". The transmission of information is from DNA to RNA to protein, or from RNA to DNA to RNA to protein. The genetic information comes from the DNA or RNA. However, the genetic information can be changed during the transmission. Previous studies showed that RNA can be modified or changed, known as RNA editing [1,2]. In addition to mRNA editing, miRNAs also exhibited editing [3-5]. The main editing type is adenosine (A) to inosine (I) modification [6]. Because inosine acts as guanosine during translation, A-to-I conversion in coding sequences leads to amino acid changes and often entails changes in protein function [3,6]. RNA can also be edited by deaminating cytidine $(\mathrm{C})$ to uridine (U).

Although the editing of mRNA includes well defined alteration of coding capacity, the editing of regulatory RNA molecules is less understood, but might interfere with their functionality. A recent understanding is that there is a broader role of RNA molecules than previously considered and that they are crucial contributors to cellular processes such as gene expression on transcriptional and post-transcriptional levels. Editing on those regulatory RNA molecules could modulate how effective they are in cellular processes.

\section{MATERIAL AND METHODS}

We speculate that RNA modification or editing takes place in all kinds of RNAs. To test this hypothesis, a liver tissue was obtained from a female fetus of 27 weeks delivered due to severe syndrome of high blood pressure of the mother in Tongji Hospital, Wuhan, Hubei, China. The fetus died shortly after delivery. The mother of the fetus had no other diseases than high blood pressure. Small RNA ( $\leq 200 \mathrm{nt})$ was isolated from about $50 \mathrm{mg}$ of the liver tissue using a mirVana ${ }^{\mathrm{TM}}$ miRNA isolation kit (Ambion, Austin, TX) following the manufacturer's instructions.

About $1 \mu \mathrm{g}$ of the isolated RNAs were polyadenylated using poly(A) polymerase (New England Biolabs). Then the Poly(A)-tailed small RNA was purified through phenol/chloroform extraction and ethanol precipitation. A 5' linker (5'-GGA CAC UGA CAU GGA CUG AAG GAG UAG AAA-3') was ligated to the poly(A)-tailed RNA using T4 RNA ligase (New England Biolabs) and the ligation products were recovered by phenol/ chloroform extraction followed by ethanol precipitation. Reverse transcription was conducted using the entire poly(A)-tailed RNA with primer (5'-CGC TAC GTA ACG GCA TGA CAG TG (T)24-3') and SuperScript III reverse transcriptase (Invitrogen) according to the manufacturer's instructions. The cDNA was PCR amplified using primers 5'-GGA CAC TGA CAT GGA CTG AAG GAG TA-3' and 5'-CGC TAC GTA ACG GCA TGA CAG TG-3'. After agarose gel electrophoresis and Gold View staining slices with PCR products were excised and purified, using EasyPure quick gel extraction kit (TransGen Biotech). The DNA fragments were directly cloned into pEASY-T1 vector (TransGen Biotech). Colony PCR was performed and the clones with PCR products were sequenced bidirectionally to avoid sequencing errors. The small RNA sequences were analyzed using BLAST analysis against the human genome and transcripts databases and miRBase (Figure 1). 


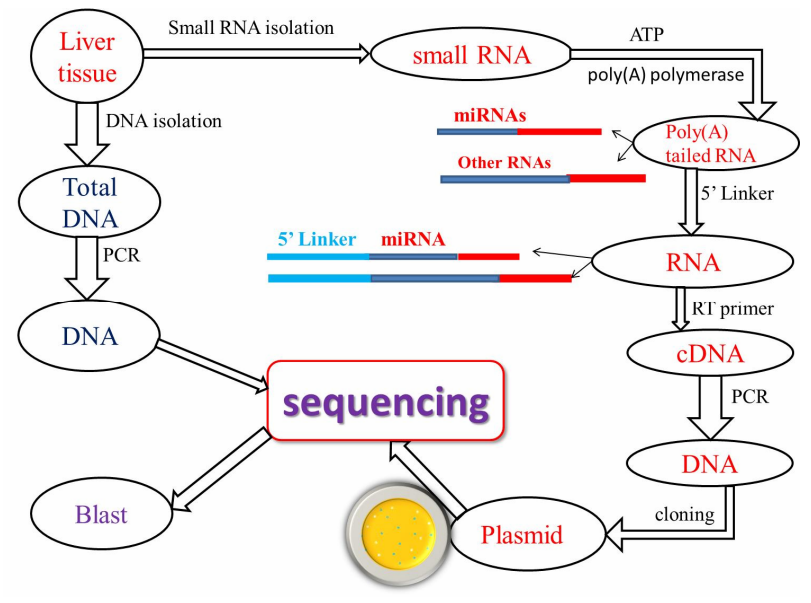

Figure 1. Workflow of the experiments performed.

\section{RESULTS AND DISCUSSION}

Several kinds of cellular RNA fragments were detected. The majority were miRNAs. Slightly more than $20 \%$ of the cloned RNAs were degraded products of abundant RNAs such as rRNA and mRNA, and few clones were unknown RNAs. RNA fragments with 1 to 2 mismatches to the sequences in the above databases were selected for SNP search. RNA editing was suggested if the possibility of being a SNP was excluded by the SNP databases search. We identified 10 changes where the RNA sequence did not match those of the corresponding DNA sequences. These changes were found in 4 miRNAs. In addition to miRNAs, $28 \mathrm{~S}$ rRNA, miscRNA (miscellaneous RNA, non-coding RNA), mRNA of SCL6A20 (Solute carrier family 6 , member 20) gene, and unknown RNA were scored to have RNA editing. We then designed primers (Table 1) to amplify the DNA regions that are transcribed to the RNAs above. DNA was isolated from the liver tissue of the female fetus. Then PCR products were bidirectionally sequenced using the $\mathrm{ABI}$ sequencer (Figure 1). Differences between DNAs and RNAs were identified in Figure 2 and Table 2. The wild types of some RNAs (hsa-mir-122, hsa-mir-451 and 28S rRNA) were also identified, suggesting that a subset of
RNAs, but not all, were edited. The hsa-mir-451 and an unknown RNA showed two types of editing respectively, indicating that there are different types of editing for one kind of RNAs. We observed six possible categories of base differences between RNA and its corresponding DNA. In addition to $A$ to $G$ changes, $T$ to $C, A$ to $C, C$ to $A, C$ to $T$, and $G$ to $T$ were also detected. There are 3 A-to-G transitions, which can be the result of deamination by ADAR or similar enzymatic activity [7]. There are 1 C-to-T transitions, which could be mediated by APOBEC or another deaminase. The 6 transversions detected cannot result from deaminase-mediated editing and the mechanism by which these differences between RNA and DNA sequences could arise is unknown.

miRNA editing may occur in the fetal stage of human liver, and additional species of RNAs could be edited. Moreover, there are several types of RNA editing emerged. Our findings support previous results on DNARNA differences in the human genome, show that these differences include miRNAs and that they are beyond A-to-G transitions. Our findings also suggest the existence of a new mechanism of RNA editing. Editing of regulatory RNA molecules might interfere with their mediated control of gene expression. Since one miRNA has the potential to regulate several genes, the editing of it might have significant contribution to the transcriptome and proteome composition. This means that the biological significance of RNA editing is broader that previously considered. The universal RNA editing may play an important role in growth and development of the fetal livers.

\section{ETHICS STATEMENT}

This research has been approved by the review board of Huazhong University of Science and Technology. We obtained tissue samples with written informed consent from the participants and parents/guardians of participants involved in the study. The ethics committee of Huazhong University of Science and Technology specifically approved the procedures.

Table 1. Primers for DNA amplification and sequencing.

\begin{tabular}{clcc}
\hline Name & \multicolumn{1}{c}{ Primer Forward } & Primer Reverse & Annealing Temperature $\left({ }^{\circ} \mathrm{C}\right)$ \\
\hline hsa-mir-23a & CCTGCTCACAAGCAGCTAAG & CTCTCTCTTTCTCCCCTCCAG & 56 \\
hsa-mir-99a & TATTAATAGGGGGCCCATGC & ATTGTTGAACGGCACTGTGT & 54 \\
hsa-mir-122 & CCCGTGATGCTTCTTTTCTC & AAAGCAAACGATGCCAAGAC & 55 \\
hsa-mir-451 & GCCTTGTTTGAGCTGGAGTC & GAGCCTGACAAGGAGGACAG & 55 \\
28s rRNA & GGCGCTAAACCATTCGTAGA & AGGAAGACGAACGGAAGGAC & 55 \\
miscRNA & CACTGAACATGGCTTTGGTG & GGTTGAGGCGTCTGTCCTAA & 57 \\
SCL6A20 mRNA & ATAGGAGAAAACCGCCCTGT & CTGTCTTAGCGAGGGGAGTG & 55 \\
unknown RNA & GGCAGCAGGTGAGAGAGAGT & GCTCTGTCTCCACCCAAATC & 61 \\
\hline
\end{tabular}




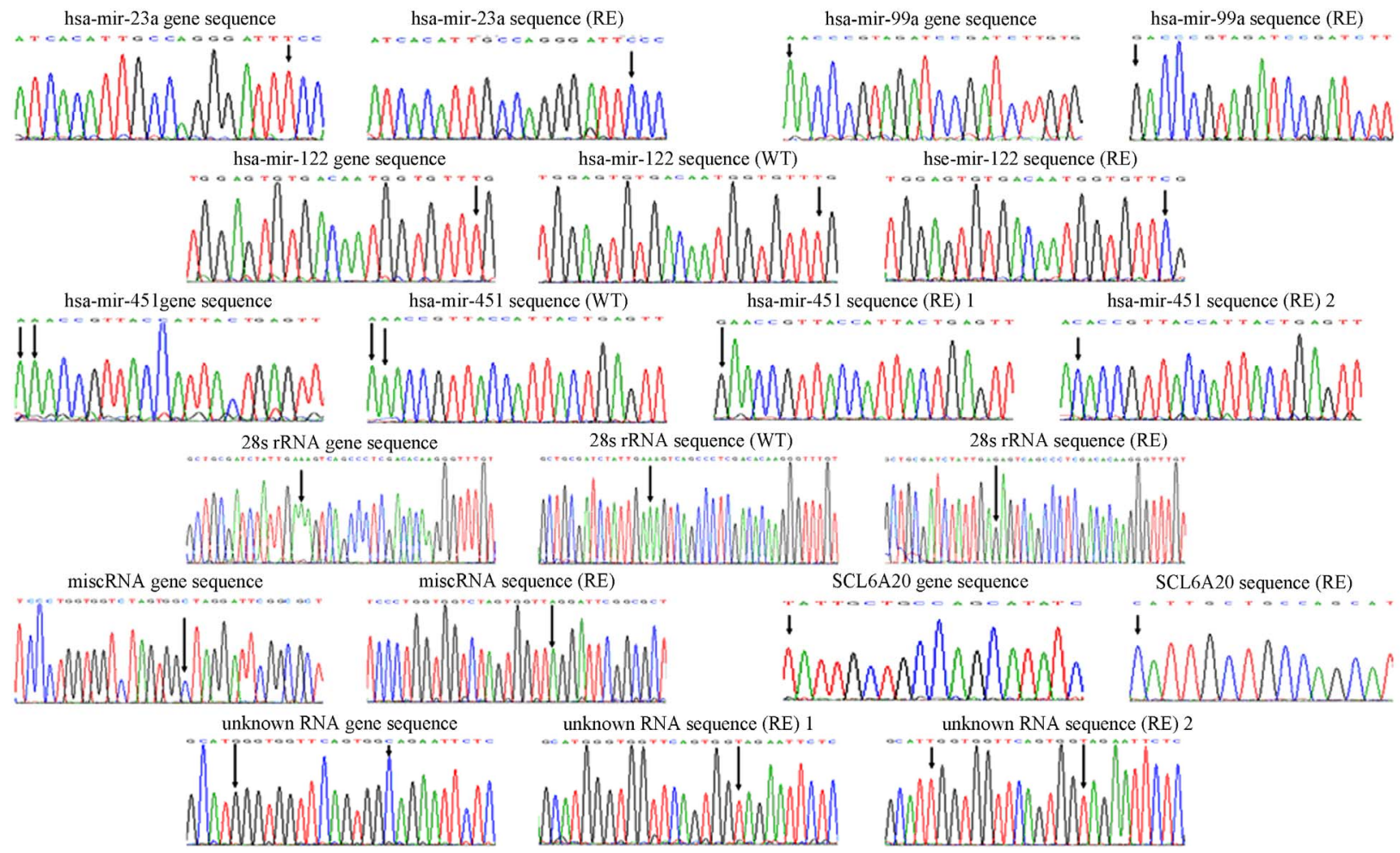

Figure 2. Comparison of sequences of DNAs and RNAs. Arrows point to base changes between DNAs and RNAs. RE, RNA editing; WT, wild type.

Table 2. RNA editing in different species of RNAs.

\begin{tabular}{|c|c|c|}
\hline RNA & Sequence & Type \\
\hline hsa-mir-23a gene & ATCACATTGCCAGGGATTTCC & \multirow{2}{*}{ T to $\mathrm{C}$} \\
\hline hsa-mir-23a(RE) & ATCACATTGCCAGGGATTCCC & \\
\hline hsa-mir-99a gene & AACCCGTAGATCCGATCTTGTG & \multirow{2}{*}{ A to $\mathrm{G}$} \\
\hline hsa-mir-99a(RE) & GACCCGTAGATCCGATCTTGTG & \\
\hline hsa-mir-122 gene & TGGAGTGTGACAATGGTGTTTG & \multirow{3}{*}{ T to $\mathrm{C}$} \\
\hline hsa-mir-122(WT) & TGGAGTGTGACAATGGTGTTTG & \\
\hline hsa-mir-122(RE) & TGGAGTGTGACAATGGTGTTCG & \\
\hline hsa-mir-451 gene & AAACCGTTACCATTACTGAGTT & \multirow{4}{*}{$\begin{array}{l}\text { A to } G \\
\text { A to } C\end{array}$} \\
\hline hsa-mir-451(WT) & AAACCGTTACCATTACTGAGTT & \\
\hline hsa-mir-451(RE)1 & GAACCGTTACCATTACTGAGTT & \\
\hline hsa-mir-451(RE)2 & ACACCGTTACCATTACTGAGTT & \\
\hline 28s rRNA gene & GCTGCGATCTATTGAAAGTCAGCCCTCGACACAAGGGTTTGT & \multirow{3}{*}{ A to $\mathrm{G}$} \\
\hline 28S rRNA (WT) & GCTGCGATCTATTGAAAGTCAGCCCTCGACACAAGGGTTTGT & \\
\hline 28S rRNA (RE) & GCTGCGATCTATTGAGAGTCAGCCCTCGACACAAGGGTTTGT & \\
\hline miscRNA gene & TCCCTGGTGGTCTAGTGGCTAGGATTCGGCGCT & \multirow{2}{*}{$\mathrm{C}$ to $\mathrm{T}$} \\
\hline miscRNA (RE) & TCCCTGGTGGTCTAGTGGTTAGGATTCGGCGCT & \\
\hline SCL6A20 gene & TATTGCTGCCAGCATATC & \multirow{2}{*}{ T to $\mathrm{C}$} \\
\hline SCL6A20 mRNA (RE) & CATTGCTGCCAGCATATC & \\
\hline unknown RNA gene & GCATGGGTGGTTCAGTGGCAGAATTCTC & \multirow{3}{*}{$\begin{array}{l}G \text { to } T \\
C \text { to } T\end{array}$} \\
\hline unknown RNA (RE)1 & GCATGGGTGGTTTAGTGGCAGAATTCTC & \\
\hline unknown RNA (RE)2 & GCATTGGTGGTTTAGTGGCAGAATTCTC & \\
\hline
\end{tabular}




\section{ACKNOWLEDGEMENTS}

This study was supported by the Huazhong University of Science and Technology (2010MS034).

\section{REFERENCES}

[1] Bass, B.L. (2002) RNA editing by adenosine deaminases that act on RNA. Annual Review of Biochemistry, 71, 817-846.

doi:10.1146/annurev.biochem.71.110601.135501

[2] Maas, S., Rich, A. and Nishikura, K. (2003) A-to-I RNA editing: Recent news and residual mysteries. Journal of Biological Chemistry, 278, 1391-1394. doi:10.1074/jbc.R200025200

[3] Daniel, J.L., Henry, M., Nicholas J.V. and Stefan, M. (2004). RNA editing of a miRNA precursor. RNA, 10,
1174-1177. doi:10.1261/rna.7350304

[4] Morse, D.P., Aruscavage, P.J. and Bass, B.L. (2002) RNA hairpins in noncoding regions of human brain and Caenorhabditis elegans mRNA are edited by adenosine deaminases that act on RNA. Proceedings of the National Academy of Sciences, 99, 7906-7911.

[5] Rueter, S.M., Dawson, T.R. and Emeson, R.B. (1999) Regulation of alternative splicing by RNA editing. $\mathrm{Na}$ ture, 399, 75-80. doi:10.1038/19992

[6] Li, M.L., Isabel, X.W., Yun, L., et al. (2011) Wide spread RNA and DNA sequence differences in the human transcriptome. Science, 333, 53-58. doi:10.1126/science.1207018

[7] Heale, B.S., Keegan, L.P., McGurk, L., et al. (2009) Editing independent effects of ADARs on the miRNA/ siRNA pathways. EMBO Journal, 28, 3145-3146. doi:10.1038/emboj.2009.244 